\title{
UNIVERSITYOF BIRMINGHAM

\section{The Contribution of Youth Sport Football to Weekend Physical Activity for Males Aged 9 to 16 Years: Variability Related to Age and Playing Position}

\author{
Fenton, Sally; Duda, Joan; Barrett, Timothy
}

DOI:

10.1123/pes.2014-0053

\section{Document Version}

Peer reviewed version

Citation for published version (Harvard):

Fenton, S, Duda, J \& Barrett, T 2015, 'The Contribution of Youth Sport Football to Weekend Physical Activity for Males Aged 9 to 16 Years: Variability Related to Age and Playing Position', Pediatric Exercise Science, vol. 27, no. 2, pp. 208-18. https://doi.org/10.1123/pes.2014-0053

Link to publication on Research at Birmingham portal

\footnotetext{
General rights

Unless a licence is specified above, all rights (including copyright and moral rights) in this document are retained by the authors and/or the copyright holders. The express permission of the copyright holder must be obtained for any use of this material other than for purposes permitted by law.

- Users may freely distribute the URL that is used to identify this publication.

- Users may download and/or print one copy of the publication from the University of Birmingham research portal for the purpose of private study or non-commercial research.

- User may use extracts from the document in line with the concept of 'fair dealing' under the Copyright, Designs and Patents Act 1988 (?)

- Users may not further distribute the material nor use it for the purposes of commercial gain.

Where a licence is displayed above, please note the terms and conditions of the licence govern your use of this document.

When citing, please reference the published version.

Take down policy

While the University of Birmingham exercises care and attention in making items available there are rare occasions when an item has been uploaded in error or has been deemed to be commercially or otherwise sensitive.

If you believe that this is the case for this document, please contact UBIRA@lists.bham.ac.uk providing details and we will remove access to the work immediately and investigate.
} 


\title{
The Contribution of Youth Sport Football to Weekend Physical Activity for Males Aged 9 to 16 Years: Variability Related to Age and Playing Position
}

\author{
ARTICLE in PEDIATRIC EXERCISE SCIENCE · NOVEMBER 2014
}

Impact Factor: $1.45 \cdot$ DOI: 10.1123/pes.2014-0053 · Source: PubMed

CITATIONS

2

3 AUTHORS, INCLUDING:

\section{Sally A.M. Fenton}

University of Birmingham

9 PUBLICATIONS 14 CITATIONS

SEE PROFILE
Joan L. Duda

University of Birmingham

332 PUBLICATIONS 9,082 CITATIONS

SEE PROFILE 
1 Doi: http://dx.doi.org/10.1123/pes.2014-0053

2 Original article available: http://journals.humankinetics.com/pes

3

5 Sally A. M. Fenton ${ }^{1}$, Joan L. Duda ${ }^{1}$, Timothy Barrett ${ }^{2}$

$6{ }^{1}$ School of Sport, Exercise and Rehabilitation Sciences, University of Birmingham,

7 Edgbaston, B15 2TT, UK.

$8{ }^{2}$ School of Clinical and Experimental Medicine, University of Birmingham, Edgbaston, 9 B152TT, UK.

10 Corresponding Author: Sally Fenton, School of Sport, Exercise and Rehabilitation

11 Sciences, University of Birmingham, Edgbaston, UK, B15 2TT. Email:

12 


\section{Abstract}

3 The aims of this study were (1) to determine minutes of moderate-to-vigorous

4 physical activity (MVPA) and vigorous physical activity (VPA) accrued in youth sport

5 football (also internationally referred to as soccer), and the contribution towards daily

6 weekend MVPA and VPA for males aged 9 to 16 years, and (2) to investigate variability in

7 these outcomes related to age and playing position. One hundred and nine male grassroots

8 footballers (Mean age $=11.98 \pm 1.75$ years) wore a GT3X accelerometer for 7 days.

9 Weekend youth sport football participation and playing position were recorded. Youth sport football MVPA $(M=51.51 \pm 17.99)$ and VPA $(M=27.78 \pm 14.55)$ contributed $60.27 \%$ and 70.68\% towards daily weekend MVPA and VPA, respectively. Overall, $36.70 \%$ of participants accumulated $\geq 60$ minutes MVPA and $69.70 \%$ accrued $\geq 20$ minutes of VPA during youth sport. For participants aged 13 to16 years, youth sport football MVPA and VPA were significantly higher, and contributed a greater amount towards daily weekend MVPA and VPA than for participants aged 9 to 12 years $(p=<.01)$. Youth sport football is an important source of MVPA and VPA at the weekend for male youth, and particularly for adolescents. Participation may offer opportunity for weekend engagement in VPA towards health enhancing levels. 


\section{Introduction}

3

As a result of the escalating global childhood obesity epidemic, considerable attention has been drawn towards the area of physical activity (PA) promotion among youth. Evidence based guidelines state that children and adolescents should engage in $\geq 60$ minutes of moderate to vigorous physical activity (MVPA) every day to accrue benefits to health $(3 ; 15 ; 23 ; 49)$. Due to recent studies indicating a dose-response relationship between PA intensity and health outcomes $(14 ; 44)$, the latest guidelines have incorporated a recommendation for vigorous physical activity (VPA), advising participation in VPA on at least three days a week for school-aged youth $(15 ; 49)$. However, concerning the volume of VPA that should be accumulated, recommendations are lacking. Indeed, little empirical evidence exists to inform guidelines for daily accumulation of VPA $(21 ; 28)$. The Healthy

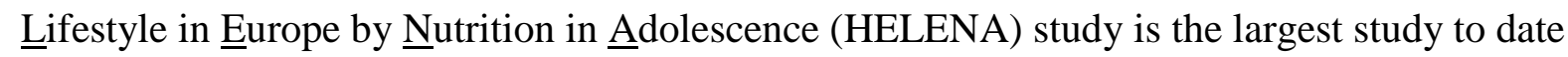
that has sought to determine the duration of daily VPA engagement likely to lead to health benefits among children and adolescents. Results from this study demonstrated approximately 18 minutes of VPA discriminated between normal weight and overweight/obesity in over 2000 European youth (Females $=10$ minutes, males $=18$ minutes) (28). Accordingly, daily recommendations for VPA were proposed at 10 and 20 minutes for female and male children and adolescents respectively.

Numerous population-based studies indicate few children and adolescents engage in recommended levels of MVPA, and daily levels of VPA are reported to be low $(9 ; 14 ; 45 ; 46)$. As a result increased efforts have been directed towards determining settings likely to be effective towards increasing engagement in MVPA and VPA among youth. The majority of research in this domain has focused upon opportunities offered within the school setting (26;38). In particular, Physical Education (PE) , active transport and school breaks (i.e., 
1 recess or lunchtime/break time) have received considerable attention, with numerous studies

2 investigating the contribution of MVPA accumulated in these environments towards

3 guidelines for MVPA and total daily MVPA and VPA (18;29;39). Recently, research has also

4 examined the extent to which MVPA and VPA accrued within different school PA settings

5 contributes differential amounts for children of different ages and for males and females

6 (29;39). However, to date, youth PA settings outside the school environment that are likely to

7 offer opportunity for regular engagement in MVPA and VPA (i.e., both weekday and

8 weekend) have been relatively overlooked.

$9 \quad$ Youth sport as an opportunity for PA engagement

10 Government organisations across the globe have advocated youth sport as a potential

11 vehicle through which levels of PA might be increased among youth $(7 ; 10 ; 35)$. However, few studies have actually examined the opportunity offered by the youth sport setting for engagement in PA. Further, a paucity of studies have employed objective measures of PA (e.g., accelerometers) to determine levels of participation in health enhancing PA during youth sport (33). The few accelerometer-based studies that have been conducted within this domain have primarily examined the opportunity offered by youth sport for engagement in MVPA towards recommended levels $(20 ; 25 ; 41 ; 51)$. Results have revealed that whilst youth sport offers the opportunity to accrue substantial amounts of MVPA, most participants are unlikely to meet daily MVPA guidelines during their involvement in youth sport $(20 ; 25 ; 41)$. To our knowledge, no studies have examined the opportunity provided by youth sport for participation in levels of VPA that have demonstrated associations with health. Given that past research indicates VPA may be more strongly linked to health outcomes than moderate PA $(14 ; 44)$, research determining the role of youth sport for encouraging engagement in health enhancing levels of VPA is warranted. Existing studies have also neglected to examine the degree to which youth sport may offer a source of both MVPA and VPA at the 
weekend. The importance of finding opportunities to increase weekend engagement in MVPA, and particularly VPA has been emphasised (45). Certainly, relative to weekday PA participation, weekend PA engagement is reported to be lower among youth $(9 ; 31)$.

Determining the settings in which youth are likely to accumulate health enhancing PA at the weekend is therefore important from a public health standpoint. Indeed, the nature of youth sport means weekend participation is likely. By contrast, research centred within the school environment will result in a focus towards weekday participation in PA.

Previous investigations examining levels of PA engagement within the youth sport context have indicated that this setting may offer differential opportunity for engagement in health enhancing PA depending on a multitude of potential determinants. For example, participation in MVPA and VPA during youth sport has been shown to vary as a function of sex, weight status (i.e., BMI), context (i.e., structure, training sessions versus match play), coach behaviour, age and sport type $(8 ; 20 ; 25 ; 41 ; 51)$. It has been suggested that the association between sport type and youth sport PA engagement may result from the differing physiological demands of specific sports (51). A similar consideration may also be relevant within sports, whereby the skill position (e.g., striker, midfielder) of some players may require participants to engage in higher levels of MVPA and VPA relative to their teammates (e.g., goal keepers). As such, where a diversity of roles is apparent within a sport, participation may provide disparate opportunities for engagement in health enhancing PA (4). However, existing studies have neglected to examine variability in youth sport PA engagement as related to different playing positions within specific sports.

Age is also a particularly pertinent factor to consider when examining the opportunity offered by youth sports for engagement in health enhancing PA. Evidence of an age related decline in PA participation among youth emphasises the importance of identifying settings that may encourage engagement in MVPA and VPA as children transition into adolescence 
1 (31;42). Adolescent youth may rely more on structured opportunities for PA engagement,

2 relative to their younger counterpart who engage in more unorganised play, or 'free play'

3 throughout the day $(1 ; 34)$. Youth sport may offer one such organised PA context through

4 which adolescents can accrue substantial amounts of health enhancing PA. To date, only one

5 study has examined age differences with regards to the accumulation of PA during youth

6 sport. Leek et al., (2011), reported younger participants (aged 7 to 10 years) engaged in

7 higher levels of MVPA and VPA during youth sport football and softball/baseball than their

8 older counterparts (aged 11 to 14 years). However, associations between age and variability

9 in youth sport VPA were not examined. Further, this investigation employed age-dependent

10 cut-points to estimate PA intensity (19), which have been shown to misclassify light PA as

11 moderate PA in children $\leq 10$ years old (47).

Extending the existent literature, the primary aims of the present study were two-fold.

First, we determined levels of MVPA and VPA accrued during youth sport football (also

internationally referred to as soccer) and the contribution made towards daily weekend

MVPA and VPA for males aged 9 to 16 years. The number of youth football participants meeting recommended guidelines for MVPA ( $\geq 60$ minutes) and engaging in levels of VPA identified as preventing excess adiposity among youth ( $\geq 20$ minutes) (27) were also determined. The second aim was to examine variability in these outcomes in relation to participant age and playing position. Football was the sport focused on in the present study due to high rates of participation across the globe, and potential application of findings to large numbers of youth sport participants. Indeed, over 21 million children and adolescents participate in youth sport football across the world (24), and research has indicated football to be among the most popular youth sports internationally $(2 ; 32)$. 
Method

3

4

5

6

7

8

9

\section{Recruitment}

Participants were a subsample of male youth sport football players primarily recruited within a larger multi-method European trial (the Promoting Adolescent Physical Activity (PAPA) Project; www.projectpapa.org)(16). Objective PA data (i.e., PA assessed via accelerometer) were collected from a subsample of participants recruited to the larger project in France, England and Greece $(\mathrm{N}=417)$. The full protocol for the PAPA Project and the core objective PA measurement protocol are detailed elsewhere (16;50). The following sections outline the protocol followed in England where additional measures were included (i.e., PA diaries).

\section{Recruitment protocol}

The PAPA project was supported by the lead football associations in each country. To aid recruitment, the English Football Association (FA) sent out regional emails to registered football clubs. Clubs were also approached to take part in the project directly by members of the research team (via phone call and/or email, $\mathrm{N}=58$ ). Interested coaches were provided with information about the study protocol and passed this information on to parents and players. A total of 46 clubs ( 36 male, 2 female and 8 mixed) were willing to participate and were recruited to the English arm of the PAPA project.

Once recruited to the larger PAPA trial, individual teams (male, $\mathrm{N}=111$, female, $\mathrm{N}=$ 23) were provided with information pertaining to an additional protocol that involved measurement of PA via accelerometer for 7 days (50). The present study utilises accelerometer data collected in a sub-sample of male English PAPA participants willing to undertake these extra measures (teams, $\mathrm{N}=38$, participants, $\mathrm{N}=149$ ). Only male footballers were recruited as a result of low female interest in participating in the accelerometer protocol 
1 (teams, $\mathrm{N}=1$ ). An additional 11 male teams (participants, $\mathrm{N}=35$ ) were recruited external to

2 the PAPA project to undertake the accelerometer protocol in order to increase the sample size

3 for the present analyses (total participants, $\mathrm{N}=184$ ). Informed consent and assent was

4 obtained from all parents and players before participating in the study. The present research

5 was approved by the local National Health Service Ethics Committee.

\section{The present study}

$7 \quad$ Participants and protocol

Participants were 184 males aged 9 to 16 years (teams, $\mathrm{N}=49$ ) who regularly

9 participate in youth sport football at the grassroots level. Grassroots football is defined as

10 participation at the recreational level, i.e., players are not playing for professional clubs or in

11 regional, national or international teams. Typically, players participating at the recreational level engage in training sessions and/or match play within an organised setting $\geq$ once per week.

A trained researcher visited football training sessions to carry out the procedures.

During the session, participants' height and weight were measured and accelerometers and

PA diaries distributed. Where possible, four players representing different playing positions

(i.e., a striker, defender, midfielder and goalkeeper) were recruited from each team.

Participants were asked to wear the accelerometer for 7 days. Researchers returned one week later to collect accelerometers and PA diaries.

\section{Measures}

\section{Anthropometrics}

Height and weight were measured with participants bare foot and wearing light clothing (e.g., shorts and t-shirt). Height was measured to the nearest $0.1 \mathrm{~cm}$ using a portable stadiometer (SECA, Leicester height measure). Weight was measured to the nearest $0.1 \mathrm{~kg}$ using electric scales (Tanita, SC3310). All measures were conducted in duplicate. Average 
1 values were calculated for height and weight and used to determine BMI [weight ( $\mathrm{kg}) /$ height

$\left.2\left(\mathrm{~m}^{2}\right)\right]$.

3

4

5

6

Physical activity

The GT3X accelerometer (Actigraph; Pensacola, FL) was used to measure MVPA and VPA. Actigraph accelerometers are the most commonly used accelerometers in PA research and have been validated against criterion measures of PA youth $(12 ; 48)$.

Accelerometers were initialised to measure PA in 15 second epochs. Verbal instructions were given by the researcher on how the accelerometer should be worn and a demonstration given.

Participants were asked to wear the accelerometer on the right hip for seven days during all waking hours, removing only for participation in water sports (e.g., swimming), bathing and sleeping. Accelerometer log sheets and PA diaries were also distributed to enable participants to record non-wear time and to aid with data cleaning and interpretation. Participants were asked to report times they had participated in youth sport football on Saturday and/or Sunday in PA diaries and to record their playing positions (i.e., striker, midfielder, defender, goalkeeper). Log sheets, PA diaries and accelerometers were collected one week later.

\section{Data processing}

Physical activity data were downloaded from the GT3X to a computer and analysed using the Actilife software (version 6.2, Actigraph). Data were checked for spurious values and periods of non-wear. Non-wear time was determined by identifying strings of consecutive zeros in the movements counts recorded by the accelerometer for $>30$ minutes, allowing for 1 minute of counts <100 (6). Cut-points derived by Evenson and colleagues were used to determine time spent in MVPA $(\geq 2296 \mathrm{cpm})$ and VPA ( $\geq 4012 \mathrm{cpm})$ (17). Youth sport and daily weekend physical activity

Time spent in youth sport football at the weekend was identified from PA diaries (i.e., day and time). For each participant, diary entries were compared against graphed data to 
1 check for accuracy in self-reported timings. Following this, individual time filters were

2 applied to the Actilife software for the identified time period to determine minutes in MVPA and VPA during youth sport football. Total daily MVPA and VPA (minutes) for the day on

which youth sport was participated in (i.e., a Saturday or Sunday) were calculated to represent daily weekend MVPA and VPA. A weekend day was considered valid where $\geq 8$ hours of wear time had been recorded.

\section{Protocol compliance}

Participants were required to have recorded one valid weekend day of accelerometer data (inclusive of their sport participation) to be retained for subsequent analyses. Of the original 184 participants, $15.8 \%(\mathrm{~N}=29)$ did not record valid daily weekend PA data on the day on which youth sport was participated in, and 9.2\% $(\mathrm{N}=17)$ failed to return completed PA diaries. A further $15.8 \%(\mathrm{~N}=29)$ did not record participation in youth sport at the weekend (e.g., monitor was removed for youth sport participation, youth sport session was cancelled) and/or information given in diaries was deemed to be inaccurate based on visual comparisons with graphed data. The final sample therefore included 109 males aged 9 to 16 years $($ compliance $=58.6 \%)$. This is comparable to compliance levels reported in the Health Survey for England 2011 for boys aged 8 to 15 years (i.e., 45-49\%) (22).

\section{Statistical analysis}

The contribution of youth sport to daily weekend MVPA and VPA

Descriptive statistics [( $\mathrm{M} \pm \mathrm{SD}$ and ranges (minutes)], for youth sport MVPA and VPA were calculated. Percentages of youth sport session time engaged in MVPA and VPA were also computed. The number of participants meeting recommended guidelines for MVPA (i.e., $\geq 60$ minutes) and achieving $\geq 20$ minutes of VPA were determined. The guideline of $\geq 20$ minutes VPA was employed based on recommendations derived from the HELENA study in which data was analysed from over European 2000 youth. Youth sport 
MVPA and VPA (minutes) were used to calculate the contribution (\%) of weekend youth sport football towards daily weekend MVPA and VPA [(Weekend youth sport MVPA/VPA

(mins)/ daily weekend MVPA/VPA) x100]. Data was checked for normality and non-

normally distributed data was log transformed. Where transformations reduced data

skewness, transformed variables were used in subsequent analysis ${ }^{1}$

\section{Variability related to age and playing position}

Descriptive statistics were calculated for youth sport MVPA and VPA and daily weekend MVPA and VPA for different playing positions (strikers $(\mathrm{N}=25)$, midfielders $(\mathrm{N}=$ $41)$, defenders $(\mathrm{N}=32)$ and goal keepers $(\mathrm{N}=6)$ and age groups [aged 9 to 12 years, $\mathrm{N}=69$ $(M=10.72, S D=.95)$, and 13 to 16 years, $N=40(M=13.98, S D=.83)]$. Age groups were devised based on data demonstrating the age related decline in PA is steepest between the ages of 13 to18 years (42). Variability in youth sport MVPA and VPA (minutes and percentage of session time) and daily weekend MVPA and VPA related to participant age and playing position were examined via ANCOVAs. Youth sport context (training versus match) was included as a covariate in all ANCOVAs ${ }^{2}$. Where total minutes in MVPA and VPA were dependent variables, youth sport session length was also included as a covariate. Chi square tests were conducted to determine if participants were more likely to meet guidelines for MVPA and VPA during youth sport football based on age group and playing position. ANCOVAs controlling for youth sport context and youth sport session length were also

\footnotetext{
${ }^{1}$ Transformations did not reduce data skewness for 2 variables; the contribution of youth sport to daily weekend MVPA (1), and VPA (2). Non-transformed variables were therefore used in subsequent analysis (Figure 1). Non-parametric statistical tests (i.e., Mann-Whitney tests) were conducted and confirmed results from parametric analysis (i.e.,ANCOVAs). Results from non-parametric tests are therefore reported in order to demonstrate effect sizes. ${ }^{2}$ Due to the nature of youth sport, participants reported engaging in a mixture of training sessions and match play across the weekend (training, $\mathrm{N}=49$ match play $=60$ ). Age was not significantly different across contexts $(t(107)=-.75, p=.46$, training $\mathrm{M}=11.77 \pm 1.73$, match play $=12.03 \pm 1.89)$, and there was no significant association between age and context $\left(\chi^{2}(1)=3.14 p=.07\right.$, Cramers $\left.\mathrm{V}=<.17\right)$.
} 
carried out to determine if the contribution of weekend youth sport to daily weekend MVPA and VPA differed significantly between age groups and playing positions.

\section{Results}

\section{Participant characteristics}

Physical characteristics by sample (total, included and excluded) are displayed in

Table 1. Independent samples t-tests demonstrated excluded participants did not differ significantly from those included with respect to age, height, weight or BMI-SDS (all $p=$ >.05). Questionnaire responses for participant ethnicity were incomplete (non-respondents; total sample $=20.65 \%$, included $=11.41 \%$, excluded $=9.24 \%]$. Among all respondents, $72.8 \%$ were white, $14.3 \%$ were Asian, $9.5 \%$ were black and $3.4 \%$ were multi-racial. Chi square tests demonstrated the ethic distribution of participants included in the final sample differed significantly from those excluded $\left(\chi^{2}(3)=17, p=<.01\right.$, included: white $=83.0 \%$, Asian $=5.7 \%$, black $=6.8 \%$, multi-racial $=4.5 \%$, excluded : white $=56.9 \%$, Asian $=27.6 \%$, black $=13.8 \%$, multi-racial $=1.7 \%$.

\section{MVPA and VPA engagement during youth sport football}

Weekend youth sport football session time was between 60 and 160 minutes $(\mathrm{M}=$ 106.74 \pm 18.90 ). Total minutes and percentage of session time engaged in MVPA and VPA during weekend youth sport football are reported in Table 2. Ranges for youth sport MVPA and VPA were 77.0 and 63.75 minutes and $71.33 \%$ and $52.54 \%$, respectively. Results demonstrated $36.70 \%$ of participants met recommended guidelines for MVPA during youth sport and $69.70 \%$ of participants' accrued $\geq 20$ minutes of VPA.

\section{The contribution of youth sport football to daily weekend MVPA and VPA}

Daily weekend MVPA and VPA were $\mathrm{M}=91.26 \pm 35.44$ and $\mathrm{M}=40.89 \pm 20.73$ respectively. MVPA accrued during weekend youth sport football contributed $60.27 \% \pm$ 
$1 \quad 19.48 \%$ (range $=25.31 \%-91.73 \%$ ) towards daily weekend MVPA. For VPA, minutes

2 accrued contributed $70.68 \% \pm 21.53 \%$ (range $=24.55 \%-100 \%)$ towards daily weekend

3

4

5

6

7

8

VPA (Figure 1).

\section{Variability related to age and playing position}

Significant differences were found for time spent in weekend youth sport MVPA and VPA between age groups. Children in the older age group engaged in significantly more minutes of $\operatorname{MVPA}\left(F(1,105)=15.76, p=<.01, \eta^{2}=.13\right)$ and $\operatorname{VPA}(F(1,105)=23.72, p=$ $\left.<.01, \eta^{2}=.18\right)$, and spent a higher percentage of youth sport session time in MVPA $(F(1$, $\left.106)=14.11, p=<.01, \eta^{2}=.12\right)$ and $\operatorname{VPA}\left(F(1,106)=21.13, p=<.01, \eta^{2}=.13\right)$ than those in the younger age group (Table 2). Chi square tests indicated older participants were more likely to accrue $\geq 60$ minutes MVPA $\left(\chi^{2}(1)=25.81, p=<.01\right.$, Cramers $\left.\mathrm{V}=.49\right)$, and $\geq 20$ minutes of VPA during youth sport $\left(\chi^{2}(1)=6.99, p=.01\right.$, Cramers $\left.\mathrm{V}=.25\right)$.

Daily weekend MVPA was not significantly different between age groups (9 to 12 years, $\mathrm{M}=90.19 \pm 33.48$ vs. 13 to 16 years, $\mathrm{M}=93.11 \pm 38.98, F(1,105)=.02, p=.89, \eta^{2}$ $=.00)$. However, daily weekend VPA was significantly higher for older compared to younger participants ( 9 to 12 years, $M=36.43 \pm 17.35$ vs. 13 to 16 years, $M=48.59 \pm 23.85, F(1$, $\left.105)=5.20, p=<.05, \eta^{2}=.05\right)$. Weekend youth sport PA contributed a significantly greater amount towards daily weekend MVPA and VPA for older compared to younger participants (Figure 1, MVPA, $F(1,105)=14.18, p=<.01, \eta^{2}=.12, \operatorname{VPA}, F(1,105)=13.14, p=<.01$, $\left.\eta^{2}=.11\right)$.

No significant differences were reported between playing positions for minutes in youth sport MVPA $\left(F(3,98)=.41, p=.75, \eta^{2}=.01\right)$ or $\operatorname{VPA}\left(F(3,98)=.58, p=.63, \eta^{2}=\right.$ $.02)$ and percent session time in $\operatorname{MVPA}\left(F(3,99)=.58, p=.65, \eta^{2}=.02\right)$ or $\operatorname{VPA}(F(3,99)$ $\left.=.62, p=.61, \eta^{2}=.02\right)$. Analyses were repeated to adjust for the significant association between age and youth sport MVPA and VPA and remained non-significant (all $p=>$. 38). 
1 There was no significant association between playing position and meeting guidelines for

$2 \operatorname{MVPA}\left(\chi^{2}(3)=.20, p=.98\right)$ or accruing $\geq 20$ minutes VPA, $\left(\chi^{2}(3)=.52, p=.92\right)$ during 3 youth sport.

Daily weekend MVPA and VPA were not significantly different between playing positions (MVPA, striker, $\mathrm{M}=95.39 \pm 36.97$, midfielder, $\mathrm{M}=90.32 \pm 32.06$, defender, $\mathrm{M}=$ $91.19 \pm 42.41$, goal keeper, $\mathrm{M}=79.29 \pm 16.67, F(3,98)=.46, p=.71, \eta^{2}=.01 ; \mathrm{VPA}$, striker, $M=40.18 \pm 19.47$, midfielder, $M=41.16 \pm 18.63$, defender, $M=42.16 \pm 25.99$, goal keeper, $\left.\mathrm{M}=32.38 \pm 9.77, F(3,98)=.63, p=.60, \eta^{2}=.02\right)$. Results also indicated weekend youth sport did not contribute significantly different amounts towards daily weekend MVPA and VPA for participants in different playing positions (Figure 1, MVPA, $F(3,98)=.53, p=$ $.67, \eta^{2}=.02$, VPA, $F(3,98)=.53, p=.67, \eta^{2}=.02$, after adjustment for age, all $\left.p=>.52\right)$.

\section{Discussion}

The present study examined levels of engagement in MVPA and VPA during youth sport football sessions and determined the contribution made towards daily weekend MVPA and VPA in males aged 9 to 16 years. Further, variability in both youth sport PA and the contribution towards daily weekend PA was investigated relative to participant age and playing position. Results demonstrate youth sport football is an important source of MVPA and VPA at the weekend for young males. In particular, participation may offer the opportunity for weekend engagement VPA towards health enhancing levels. Findings also revealed differences between children (aged 9 to 12 years) and adolescents (aged 13 to 16 years) in terms of their levels of engagement in MVPA and VPA during youth sport, and the contribution made towards daily weekend engagement in these behaviours.

MVPA and VPA engagement during youth sport football

Present results demonstrated that on average, male youth sport football participants engaged in approximately 52 and 28 minutes of MVPA and VPA, respectively. As such, 
1 findings indicate that participation in weekend youth sport football may encourage engagement in MVPA and VPA towards levels identified as being beneficial for health (23;28). Leek et al., (2011) reported comparable levels of MVPA and VPA participation during youth sport among boys aged 7 to 14 years. Specifically, results indicated softball/baseball and soccer (football) participants accumulated 56 minutes of MVPA and up to 30 minutes of VPA during youth sport. However, other studies have reported lower estimates of youth sport MVPA and VPA engagement among boys of similar ages (41;51). For example, Wickel et al., (2007) found boys participating in basketball, flag football and soccer (football) aged 6 to 12 years to accumulate 26 minutes of MVPA and up to 18 minutes of VPA minutes during youth sport time. Similarly, Sacheck et al., (2011) reported boys, aged 7 to 10 years, accrued only 16 minutes of MVPA and 4 minutes of VPA during youth sport football.

Differing results across studies may partly reflect sample differences in sport type. For example, Leek et al., (2011) reported male footballers (soccer participants) accrued approximately 56 of MVPA during youth sport, whereas male softball/baseball participants participated in MVPA for approximately 48 minutes $^{3}$. However, the variability in levels of MVPA and VPA reported may, in part, also be due to differences between studies in terms of youth sport session length. Indeed, after accounting for youth sport time, Wickel et al., (2007) and Leek et al., (2011) reported boys representing a variety of different sports, spent on average $49 \%$ and $54 \%$ of youth sport time engaged in MVPA, respectively. In the present study, male youth sport footballers were engaged in MVPA for 50\% of their youth sport session. In addition, Wickel et al., (2007) demonstrated VPA was participated in for $22 \%$ of youth sport time, and current results revealed youth sport footballers spent $27 \%$ of youth sport sessions engaged in VPA.

\footnotetext{
${ }^{3}$ Data taken from graph (Leek et al., 2011)
} 
Whilst disparate study findings may indeed reflect sample differences related to sport

2 type and youth sport session length, the findings of Sacheck et al., (2011) also point to the need to consider the data reduction procedures when drawing comparisons across investigations. Their study revealed boys accrued only 17 minutes, and spent $30 \%$ of youth sport time engaged in MVPA, estimates considerably lower than reported in both past studies and the present research (25;51). Both Wickel et al., (2007) and Leek et al., (2011) used agedependent accelerometer cut-points to estimate engagement in MVPA and VPA. Conversely, Sacheck, et al., (2011) employed single value cut-points to measure MVPA and VPA in participants aged 7 to 10 years $[\geq 3180-8159 \mathrm{cpm}$ (MVPA), $\geq 8160 \mathrm{cpm}$ (VPA)]. These cutpoints are relatively higher than the equivalent age-dependent cut-points for youth of the same age $[\geq 705-1017 \mathrm{cpm}(\mathrm{MVPA}), \geq 3136-3696 \mathrm{cpm}(\mathrm{VPA})]$. Thus, the more conservative cut-points utilized by Sacheck et al., (2011) may have contributed towards the lower levels of MVPA and VPA reported in their study.

The present study employed the Evenson et al., (2008) cut-points to estimate MVPA and VPA engagement during youth sport. These single-value cut-points have been reported to demonstrate superior reliability and validity over other independently validated cut-points for youth and are currently recommended for use in children and adolescents (47). Present findings may therefore more accurately reflect frequency, intensity and duration of PA engagement among male youth sport football participants than those reported in some previous studies $(25 ; 41 ; 51)$. To our knowledge, only one study to date has employed the Evenson et al., (2008) cut-points to examine levels of MVPA engagement during youth sport football (8). Cohen et al., (2014) revealed participants accrued only 23 minutes of MVPA during youth sport football and spent $37 \%$ of youth sport time engaged in MVPA. The lower levels of MVPA, relative to those reported presently, may be partly due to the inclusion of 
1 female participants in the previous study. Certainly, past research has demonstrated girls to engage in significantly lower levels of MVPA during youth sport than boys $(20 ; 25)$. To our knowledge, ours is the first investigation to examine the opportunity provided by youth sport for engagement in levels of VPA that have demonstrated associations with health. Results revealed $70 \%$ of participants accrued $\geq 20$ minutes of VPA, a cut-off reported to discriminate between normal weight and overweight/obesity in male European youth (28). Thus, participation in youth sport football may be conducive towards engagement in levels of VPA required to prevent the development of excess adiposity in young male footballers. However, whilst 20 minutes of VPA has been found to confer positive health benefits, this cut-off is based on the results of a single study. Hay et al., (2012) reported independent associations between vigorous PA and cardiometabolic risk factors occurred across a narrow range of PA duration, revealing only 7 minutes of VPA to be associated with reduced risk for overweight and elevated systolic blood pressure among Canadian youth (21). In the current study, $98.17 \%$ of participants $(\mathrm{N}=107)$ accrued $\geq 7$ minutes of VPA during youth sport (data not shown $)^{4}$. Additional research is therefore necessary to determine levels of VPA participation which are associated with benefits across a range of health indicators (e.g., vascular, metabolic) to inform evidence-based guidelines for VPA. The development of such guidelines will facilitate researchers in their attempts to determine youth PA settings likely to offer children and adolescents the optimal opportunity for engagement in levels of VPA conducive towards enhanced health.

Whilst present findings indicate youth sport football may indeed offer opportunities for young males to accrue substantial amounts of VPA, results suggest participation in this PA context alone is unlikely to promote engagement in MVPA toward levels required to lead to health benefits. Results revealed only $37 \%$ of participants met recommended guidelines for

\footnotetext{
${ }^{4}$ Data available from the first author upon request
} 
1 MVPA during youth sport sessions, and youth sport MVPA was reported to be as low as 14

2 minutes ( $\sim 14 \%$ of session time). Whilst the use of differing accelerometer cut-points somewhat precludes comparisons across studies, current findings are in line with those of

4 Leek et al., (2011) who found only $24 \%$ of participants to meet daily guidelines for MVPA

5 during youth sport, and investigations reporting participants can spend up to $70 \%$ of youth

6 sport time engaged in PA below a moderate intensity $(20 ; 41)$. Taken together, past and

7 present findings suggest there is scope to increase the duration of engagement in MVPA to

8 further optimise youth sport participation, and in turn, contribute towards obesity and disease

9 prevention among youth active in this context. However, also important to consider is that

10 whilst a number of participants accrued $\geq 20$ minutes of VPA, nearly a third did not achieve

11 this recommendation. Moreover, only $27 \%$ of youth sport time was spent engaged in VPA.

Maximising youth sport as a context for PA engagement should therefore help to contribute towards more youth achieving recommended and health enhancing levels of both MVPA and VPA on youth sport days.

\section{The contribution of youth sport football to daily weekend MVPA and VPA}

To our knowledge, only one previous study has examined the contribution of youth sport towards objectively measured daily MVPA, reporting youth sport contributed 23\% towards MVPA on a weekday (51). The current study is the first to determine the contribution of youth sport towards daily weekend levels of MVPA, revealing a contribution of approximately $60 \%$. The higher contribution observed in the present research suggests that youth sport likely becomes a more important source of MVPA at the weekend, in the absence of structured opportunities for PA engagement that are inherent within the school day (e.g., physical education, school breaks/recess). Extant research has also not considered the contribution of youth sport to daily weekday or weekend VPA participation. 
1 Previous work has emphasised the necessity to study the main activities undertaken when engaged in VPA (45). The present study, therefore, contributes to the literature by reporting VPA accumulated during youth sport comprises approximately $71 \%$ towards daily weekend VPA. As such, results point to youth sport football as an important source of VPA at the weekend on days in which youth sport is participated in. Still, studies that examine the contribution of youth sport to weekday VPA are necessary to determine the value of PA participation in this context as a source of regular VPA throughout the week.

\section{Variability related to age and playing position}

Current findings revealed that participants, aged 13 to 16 years, accrued more minutes of MVPA and VPA, and spent a higher percentage time engaged in MVPA and VPA, during youth sport compared to those aged 9 to 12 years. Results also indicated that relative to their younger counterparts, older participants were more likely to meet guidelines for MVPA and VPA during youth sport football. Moreover, PA accumulated in this setting contributed a greater amount towards daily weekend MVPA and VPA for older participants. These results imply that youth sport participation may offer greater opportunity for engagement in MVPA and VPA, and represent a greater source of weekend PA for youth during adolescence than during childhood. Leek et al., (2011) revealed contrasting findings to those reported presently, demonstrating youth sport participants' aged 7-10 years engaged in 7 minutes more MVPA during youth sport than those aged 11-14 years. Divergent findings may be explained by the use of age-dependent cut-points in the past study and likely overestimation of MVPA in participants $\leq 10$ years old (47).

Given the dearth of studies investigating age related variability in youth sport PA, discussion as to why older participants engaged in higher level of MVPA and VPA during youth sport than younger participants are somewhat speculative. Perhaps, as children reach adolescence, the focus of the coach is removed away from knowledge delivery and 
1 instruction regarding basic skills, towards refining skills via game play and activities that

2 facilitate aerobic conditioning, in order to prepare for more competitive play. Indeed, a recent study reported youth sport MVPA to be lower when coaches were providing general

4 instruction and engaged in skill demonstration, relative to during drills, fitness instruction and

5 small group activities (8). Thus, adjustment in the organisation and focus of youth sport

6 sessions as children transition through the stages of sport specialisation (11), coupled with an

7 inherent age related increase in strength and power as youth advance through puberty (40), may result in increased engagement in high intensity PA as children progress into adolescent teams. In turn, youth sport football may likely offer a greater source of health enhancing PA for adolescents relative to children. The present study provides initial evidence for this contention, demonstrating youth sport contributed $72 \%$ and 54\% towards daily MVPA for adolescents and children respectively. Perhaps as children advance into adolescence, they rely more on organised PA settings (such as youth sport) as opportunities for engagement in health enhancing PA. For example, Nilsson et al., (2009) demonstrated frequency of outdoor play after school to be a significant correlate for daily MVPA in 9 year old European youth, which was attenuated in favour of participation in sport and exercise in clubs in 15 year olds (34). Encouraging participation in youth sport football may therefore have implications for counteracting the age related decline in PA observed among youth during the teenage years $(29 ; 30 ; 45)$. Indeed, a more marked decline in MVPA has been reported to occur between the ages of 13 and 16 in non-sport participants compared to sport participants (27). The present investigation indicated playing position was not related to variability in youth sport MVPA or VPA among male youth sport footballers. By contrast, research conducted in adults indicates position classification to be related to time spent running, sprinting, and engaged in high intensity activity $(4 ; 13 ; 30)$. However, extant studies have largely examined males participating in professional leagues at the elite level. Thus, current 
1 results suggest that where players are participating at the earlier stages of sport specialisation

2 (e.g., grassroots participation), the physical demands of different playing positions (and resulting levels of PA engagement), may be more comparable. Nevertheless, there was a

4 trend towards lower levels of MVPA and VPA among goal keepers. The lack of a significant PA differences between outfield players and goal keepers in the present study may be a consequence of the low number of goal keepers recruited and the high variability in youth sport MVPA and VPA among players.

Strengths of the present study include the use of accelerometers to measure PA, allowing estimation of duration and intensity of activity during youth sport football sessions. In addition, the broad age range of participants recruited allows important questions to be answered pertaining to the value of youth sport as a source of health enhancing PA for children, relative to adolescents. Moreover, the sample represented youth sport footballers from 49 different teams. As such, results presented are less likely confounded by betweenteam differences (e.g., coach behaviour) relative to previous studies in which fewer teams have been recruited $(8 ; 20 ; 25 ; 41)$. Limitations to the current research include possible sample bias; participants were self-selected and largely represent white English males who participate in youth sport football. Thus, results presented may not be generalisable to young people representing the wider ethnic distribution of children who participate in youth sport football, females and children and adolescents who participate in other youth sports. Indeed, studies conducted within the youth sport domain highlight the multitude of factors which can affect PA engagement during youth sport $(8 ; 20 ; 25 ; 41 ; 51)$. Thus, future studies are needed to determine the opportunity offered by specific sports for participation in health enhancing PA (and the contribution towards daily PA) across the broad spectrum of youth who participate in sport at grassroots level. In particular and in view of research demonstrating girls engage in 
1 lower levels of PA during youth sport than boys, there is a need to examine context specific

2 PA engagement in boys and girls separately (36).

collect data pertaining to pubertal stage of participants. Data were collected in small groups and as such, asking questions pertaining to stage of biological maturation (e.g., the Tanner scale) in this group setting may have reduced player's willingness to participate. However, whilst we are unable to account for the role of biological maturation when examining age related differences, a recent review concluded associations between biological maturation and levels of PA engagement among youth were inconsistent and often reported to be low (43). Moreover, this review reported chronological age independently influences the association between biological maturation and PA. Indeed, studies which sample sufficiently across age ranges have demonstrated pubertal status is typically highly correlated with age $(5 ; 37)$, which may account for this mediating effect. Thus, whilst associations between biological maturation and youth sport MVPA and VPA may indeed be plausible, this relationship is unlikely to have a significant influence on the results reported herein.

In conclusion, results demonstrate that youth sport football is an important source of MVPA and VPA at the weekend for male youth aged 9 to 16 years. Present findings also indicate that participation in youth sport football may encourage weekend engagement in VPA towards health enhancing levels. However, youth sport football may encourage higher engagement in MVPA and VPA, and represent a more important source of weekend MVPA and VPA, for older compared to younger participants. Observed variability in youth sport MVPA and VPA, among both children and adolescents active in this context, highlights the need to optimise the youth sport experience in regard to young people's participation in health enhancing PA. 
(1) Anderson SE, Economos CD, Must A. Active play and screen time in US children aged 4 to 11 years in relation to sociodemographic and weight status characteristics: a nationally representative cross-sectional analysis. BMC Public Health. 2008;8:366.

(2) Australian Bureau of Statistics. Children's Participation in Cultural and Leisure Activities [internet]. 2009. Available from:

http://www.abs.gov.au/ausstats/abs@.nsf/Products/4901.0 Apr+2009 Main+Featur $\underline{\text { es } \sim \text { Organised+sport }}$

(3) Australian Government: Department for Health and Ageing. Physical Activity Guidelines for Children (5-12 yrs) [internet]. 2004. Available from: http://www.beactive. wa.gov.au/index.php?id=474

(4) Bloomfield J, Poleman R, O'Donoghue P. Physical demands of different positions in FA Premiere League soccer. J Sports Sci Med. 2007;6(1):63-70.

(5) Booth A, Johnson DR, Granger DA, Crouter AC, McHale S. Testosterone and child and adolescent adjustment : the moderating role of parent and child relationships. Developmental Psychology. 2003;39(1):85-98.

(6) Cain K, Sallis JF, Conway TL, Van Dyck D, Calhoon L. Using Accelerometers in Youth Physical Activity Studies: A Review of Methods. J Phys Act Health. 2013;10(3):437-50. 
(7) Centers for Disease Control and Prevention. Promoting Better Health for Young People through Physical Activity and Sports [report]. 2000. Available from: http://usa.usembassy.de/etexts/sport/Promoting_better_health

(8) Cohen A, McDonald S, McIver K, Pate R, Trost S. Assessing Physical Activity During Youth Sport: The Observational System for Recording Activity in Children: Youth Sports. Pediatr Exerc Sci. 2014;26(2):203-9.

(9) Collings PJ, Wijndaele K, Corder K, Westgate K, Ridgway CL, Dunn V, et al. Levels and patterns of objectively-measured physical activity volume and intensity distribution in UK adolescents: the ROOTS study. Int J Behav Nutr Phys Act. $2014 ; 11(23)$.

(10) Commission of the European Communities. White Paper on Sport [report]. 2007. Available from: http://europa.eu/documentation/official-docs/whitepapers/index_en.htm

(11) Côté J. The influence of the family in the development of talent in sports. Sports Psycholigist. 1999;13:395-417.

(12) de Vries SI, Van Hirtum H, Bakker I, Hopman-Rock M, Hirasing RA, Van Mechelen W. Validity and Reproducibility of Motion Sensors in Youth: A Systematic Update. Med Sci Sports Exerc. 2009;41(4):818-27.

(13) Dellal A, Chamari K, Wong PL, Ahmaidi S, Keller D, Barros R, et al. Comparison of physical and technical performance in European soccer match-play: FA Premier League and La Liga. Eur J Sport Sci. 2011;11(1):51-9. 
(14) Dencker M, Andersen LB. Health-related aspects of objectively measured daily physical activity in children. Clin Physiol Funct Imaging. 2008;28(3):133-44.

(15) Department of Health. UK physical activity guidelines [internet]. 2011. Available from: http://www.dh.gov.uk

(16) Duda JL, Quested E, Haug E, Samdal O, Wold B, Balaguer I, et al. Promoting Adolescent health through an intervention aimed at improving the quality of their participation in Physical Activity (PAPA): Background to the project and main trial protocol. International Journal of Sport and Exercise Psychology. 2013;11(4):31927.

(17) Evenson KR, Catellier DJ, Gill K, Ondrak KS, McMurray RG. Calibration of two objective measures of physical activity for children. J Sports Sci. 2006;24(14):155765.

(18) Fairclough S, Stratton G. 'Physical education makes you fit and healthy'. Physical education's contribution to young people's physical activity levels. Health Educ Res. 2005;20(1):14-23.

(19) Freedson P, Pober D, Janz KF. Calibration of accelerometer output for children. Med Sci Sports Exerc. 2005;37(11 Suppl):S523-S530.

(20) Guagliano JM, Rosenkranz RR, Kolt GS. Girls' Physical Activity Levels during Organized Sports in Australia. Med Sci Sports Exerc. 2013;45(1):116-22. 
(21) Hay J, Maximova K, Durksen A, Carson V, Rinaldi RL, Torrance B, et al. Physical Activity Intensity and Cardiometabolic Risk in Youth. Arch Pediatr Adolesc Med. 2012;166(11):1022-9.

(22) Health and Social Care Information Centre. Health Survey for England, 2011: Health, social care and lifestyles. Summary of key findings [report]. 2012. Available from: www.ic.nhs.uk/pubs/hse11report

(23) Janssen I, Leblanc AG. Systematic review of the health benefits of physical activity and fitness in school-aged children and youth. Int J Behav Nutr Phys Act. 2010;7:40.

(24) Kunz M. 265 million playing football. FIFA Magazine, July issue [internet]. 2007. Available from: http://www.fifa.com/mm/document/fifafacts/bcoffsurv/emaga_9384_10704.pdf

(25) Leek D, Carlson JA, Cain KL, Henrichon S, Rosenberg D, Patrick K, et al. Physical activity during youth sports practices. Arch Pediatr Adolesc Med. 2011;165(4):2949.

(26) Lonsdale C, Rosenkranz RR, Peralta LR, Bennie A, Fahey P, Lubans DR. A systematic review and meta-analysis of interventions designed to increase moderateto-vigorous physical activity in school physical education lessons. Prev Med. 2013;56(2):152-61. 
(27) Machado-Rodrigues AmM, Coelho e Silva MJ, Mota J, Santos RM, Cummin SP, Malina RM. Physical Activity and Energy Expenditure in Adolescent Male Sport Participants and Nonparticipants Aged 13 to 16 Years. J Phys Act Health. 2012;9(5):626-33.

(28) Martinez-Gomez D, Ruiz JR, Ortega FB, Veiga OL, Moliner-Urdiales D, Mauro B, et al. Recommended Levels of Physical Activity to Avoid an Excess of Body Fat in European Adolescents: The HELENA Study. Am J Prev Med. 2010;39(3):203-11.

(29) Meyer U, Roth R, Zahner L, Gerber M, Puder JJ, Hebestreit H, et al. Contribution of physical education to overall physical activity. Scand J Med Sci Sports. 2013;23(5):600-6.

(30) Mohr M, Krustrup P, Bangsbo J. Match performance of high-standard soccer players with special reference to development of fatigue. J Sports Sci. 2003;1;21(7):519-28.

(31) Nader PR, Bradley RH, Houts RM, McRitchie SL, O'Brien M. Moderate-tovigorous physical activity from ages 9 to 15 years. JAMA. 2008;300(3):295-305.

(32) National Council of Youth Sports. Report on Trends and Participation in Organized Youth Sports [report]. 2008. Available from: http://www.ncys.org/publications/2008-sports-participation-study.php

(33) Nelson TF, Stovitz SD, Thomas M, LaVoi NM, Bauer KW, Neumark-Sztainer D. Do youth sports prevent pediatric obesity? A systematic review and commentary. Curr Sports Med Rep. 2011;10(6):360-70. 
(34) Nilsson A, Andersen LB, Ommundsen Y, Froberg K, Sardinha LB, Piehl-Aulin K, et al. Correlates of objectively assessed physical activity and sedentary time in children: a cross-sectional study (The European Youth Heart Study). BMC Public Health. 2009;9:322.

(35) NSW Department of Health. NSW Government Plan for Preventing Overweight and Obesity in Children, Young People \& their Families 2009 - 2011 [report]. 2009. Available from: http://www0.health.nsw.gov.au/pubs/2009/obesity_action_plan.html

(36) Pate RR, O'Neill JR. Youth sports programs: contribution to physical activity. Arch Pediatr Adolesc Med. 2011;165(4):369-70.

(37) Patia-Spear L. Brain development and adolescent behavior. In: Coch D, Fischer KW, Dawson G, editors. Human Behavior, Learning and the Developing Brain.New York: The Guildford Press; 2007.

(38) Ridgers ND, Salmon J, Parrish AM, Stanley RM, Okely AD. Physical activity during school recess: a systematic review. Am J Prev Med. 2012;43(3):320-8.

(39) Ridgers ND, Timperio A, Crawford D, Salmon J. Five-year changes in school recess and lunchtime and the contribution to children's daily physical activity. Br J Sports Med. 2012;46(10):741-6.

(40) Roemmich JN, Rogol AD. Physiology of growth and development. It's relationship to performance in the young athlete. Clin Sports Med. 1995;14(3):483-502. 
(41) Sacheck JM, Nelson T, Ficker L, Kafka T, Kuder J, Economos CD. Physical activity during soccer and its contribution to physical activity recommendations in normal weight and overweight children. Pediatr Exerc Sci. 2011;23(2):281-92.

(42) Sallis JF. Age-related decline in physical activity: a synthesis of human and animal studies. Med Sci Sports Exerc. 2000;32(9):1598-600.

(43) Sherar LB, Cumming SP, Eisenmann JC, Baxter-Jones ADG, Malina RM. Adolescent Biological Maturity and Physical Activity: Biology Meets Behavior. Pediatr Exerc Sci. 2010;22(3):332-49.

(44) Steele RM, van Sluijs EM, Cassidy A, Griffin SJ, Ekelund U. Targeting sedentary time or moderate- and vigorous-intensity activity: independent relations with adiposity in a population-based sample of 10-y-old British children. Am J Clin Nutr. 2009;90(5):1185-92.

(45) Steele RM, van Sluijs EM, Sharp SJ, Landsbaugh JR, Ekelund U, Griffin SJ. An investigation of patterns of children's sedentary and vigorous physical activity throughout the week. Int J Behav Nutr Phys Act. 2010;7:88.

(46) Troiano RP, Berrigan D, Dodd KW, Masse LC, Tilert T, McDowell M. Physical Activity in the United States Measured by Accelerometer. Med Sci Sports Exerc. 2008;40(1):181-8.

(47) Trost SG, Loprinzi PD, Moore R, Pfeiffer KA. Comparison of accelerometer cut points for predicting activity intensity in youth. Med Sci Sports Exerc. 2011;43(7):1360-8. 
(48) Trost SG. State of the Art Reviews: Measurement of Physical Activity in Children and Adolescents. Am J Lifestyle Med. 2007;1(4):299-314.

(49) US Department of Health and Human Services. Physical activity guidelines for Americans [internet]. 2013. Available from:

http://www.health.gov/PAguidelines/guidelines/chapter3.aspx.

(50) Van Hoye Al, Fenton S, Krommidas C, Heuzé J-P, Quested E, Papaioannou A, et al. Physical activity and sedentary behaviours among grassroots football players: A comparison across three European countries. International Journal of Sport and Exercise Psychology. 2013;11(4):341-50.

(51) Wickel EE, Eisenmann JC. Contribution of youth sport to total daily physical activity among 6- to 12-yr-old boys. Med Sci Sports Exerc. 2007;39(9):1493-500.

\section{Figure captions:}

14 Figure 1 The contribution of youth sport to daily weekend MVPA and VPA stratified by age 5 group and playing position. 